\title{
SELEÇÃO PRECOCE PARA A PRODUTIVIDADE DE GRÃOS NO FEIJOEIRO ${ }^{(1)}$
}

\author{
CARLOTA JOAQUINA DE SOUSA ROSAL ${ }^{(2)}$; MAGNO ANTONIO PATTO \\ RAMALHO $^{(2)}$; FLÁVIA MARIA AVELAR GONÇALVES ${ }^{(2)}$; ÂNGELA DE \\ FÁTIMA BARBOSA ABREU ${ }^{(3)}$
}

\begin{abstract}
RESUMO
O objetivo do presente trabalho foi avaliar a eficiência da seleção precoce para o caráter produtividade de grãos na cultura do feijão (Phaseolus vulgaris L.). Para tanto foi utilizado o cruzamento entre os cultivares Carioca $\left(\mathrm{P}_{1}\right)$ e FT- Tarumã $\left(\mathrm{P}_{2}\right)$. Foram avaliadas 192 famílias, 64 das quais derivadas de plantas $\mathrm{F}_{2}$, outras 64 de plantas $\mathrm{F}_{2}$ do $\mathrm{RC}_{1}\left(\mathrm{~F}_{1} \times \mathrm{P}_{1}\right)$ e 64 , ainda, de plantas $\mathrm{F}_{2}$ de $\mathrm{RC}_{2}\left(\mathrm{~F}_{1} \times \mathrm{P}_{2}\right)$. As famílias foram conduzidas empregando-se o método Bulk dentro de famílias $\mathrm{F}_{2}$ e avaliadas nas gerações $\mathrm{F}_{2: 3}$ (fevereiro de 1997), $F_{2: 4}$ (julho de 1997), $F_{2: 5}$ (novembro de 1997), $F_{2: 6}$ (fevereiro de 1998), na área experimental do Departamento de Biologia da Universidade Federal de Lavras (MG). Utilizou-se o delineamento látice simples 14 × 14 na avaliação das 192 famílias juntamente com os pais e duas testemunhas. A partir das análises de variância dos dados da produtividade de grãos, foram estimados os componentes da variância genética e fenotípica, a herdabilidade realizada, as estimativas de correlações entre as famílias nas sucessivas gerações e o ganho observado com a seleção divergente. Constatou-se que: as famílias de melhor desempenho foram observadas no $\mathrm{RC}_{2}$; a interação genótipos $\mathrm{x}$ ambientes afetou a seleção precoce, valendo destacar que, para melhorar sua eficiência, as famílias devem ser avaliadas no mínimo durante duas gerações; a seleção precoce foi eficiente principalmente para a eliminação das famílias com pior desempenho, reduzindo, dessa forma, o trabalho dos melhoristas em gerações avançadas.

Palavras-chave: seleção precoce, interação genótipos x ambientes, feijão.
\end{abstract}

\section{ABSTRACT \\ EARLY SELECTION FOR COMMON BEAN GRAIN YIELD}

The objective of this study was to evaluate the efficiency of the early selection of common bean (Phaseolus vulgaris L.) grain yield. 192 families from the cross 'Carioca' $\left(\mathrm{P}_{1}\right) \times$ 'FT-Tarumã' $\left(\mathrm{P}_{2}\right)$, which 64 from $\mathrm{F}_{2}$ generation and 128 from $\mathrm{F}_{2}$ of the backcrosses ( 64 of the $\mathrm{BC}_{1}$ and 64 of the $\mathrm{BC}_{2}$ ) were assessed. These families were conducted by within $\mathrm{F}_{2}$ family bulk breeding and evaluated in the generations $F_{2: 3}$ (February/1997), F2:4 (July/1997), F2:5 (November/1997) and F2:6 (February/1998), in the Department of Biology of the Federal University of Lavras, Minas Gerais State, Brazil. It was used the simple lattice design , $14 \times 14$, including the 192 families, the parents and two checks. The following genetic and phenotypic parameters were estimated: genetic and phenotypic variances; realized heritability; correlations between families of the evaluated generations; and genetic gain observed based on divergent selection. General results indicated that the best families were observed in all generations derived from $\mathrm{BC}_{2}$ and genotypes $\mathrm{x}$ environments interaction affected the early selection. Therefore, to

( $\left.{ }^{1}\right)$ Parte da dissertação da primeira autora, apresentada à Universidade Federal de Lavras (UFLA), como exigência para obtenção do grau de Mestre em Genética e Melhoramento de Plantas. Recebido para publicação em 18 de março de 1999 e aceito em 23 de maio de 2000.

$\left(^{2}\right)$ Departamento de Biologia/UFLA, Caixa Postal 37, 37200-000 Lavras (MG). E-mail: magnoapr@ufla.br

(3) EMBRAPA/EPAMIG, Caixa Postal 176, 37200-000 Lavras (MG). 
improve its efficiency, the families should be evaluated for at least two generations. The early selection was efficient mainly for elimination of the worst families, reducing in that way the work of breeder in advanced generations.

Key words: early selection, genotypes $\mathrm{x}$ environments interaction, common bean.

\section{INTRODUÇÃO}

A maioria dos caracteres de importância econômica, como a produção de grãos, são controlados por um grande número de genes. Nesse sentido, para se obterem indivíduos extremos é necessário trabalhar com grandes populações. No caso das populações segregantes de plantas autógamas, a probabilidade de manter o indivíduo com todos os alelos favoráveis é reduzida, com o avanço das gerações (RAmAlHo et al., 1993). Isso exige que se avalie um número crescente de famílias, com o decorrer das gerações, o que, muitas vezes, inviabiliza o sucesso dos melhoristas.

Para atenuar esse problema, a principal alternativa seria a seleção de plantas e/ou famílias já nas gerações iniciais, isto é, realizar a seleção precoce. Visando verificar sua eficiência, há, na literatura, alguns trabalhos que afirmam que a seleção só é efetiva para caracteres de alta herdabilidade. Contudo, devese enfatizar que, em tais trabalhos, a seleção precoce foi efetuada com base na seleção visual, a qual, por diversas vezes, tem-se mostrado ineficiente para caracteres de baixa herdabilidade (HANSON et al., 1962; PatiÑo e Singh, 1989; Cutrim, et al., 1997). Desse modo, persiste a dúvida acerca da eficácia da seleção precoce, em virtude do processo seletivo utilizado.

No método de seleção denominado bulk dentro das famílias $F_{2}$, é possível que as mesmas sejam avaliadas em experimentos com repetições, já a partir da geração $F_{2: 3}$, e a seleção precoce poderia ser, então, efetuada sem a utilização da seleção visual. Nessa condição, espera-se que a seleção precoce possa ser mais efetiva. BERNARDO (1991) demonstrou que a eficiência da seleção precoce é função da correlação entre o desempenho fenotípico da família, na geração de seleção, e o desempenho genotípico na geração avançada. Essa correlação, por sua vez, é resultado da correlação genética entre famílias, nas gerações consideradas, e a raiz quadrada da herdabilidade do caráter na geração precoce. Tendo em vista a correlação genética normalmente alta, pois é função apenas do coeficiente de endogamia, nas gerações consideradas, vale ressaltar que a eficiência da seleção precoce é dependente principalmente da herdabilidade do caráter sob seleção, nas gerações iniciais, o que está de acordo com inúmeros relatos de sua eficiência somente para caracteres de alta herdabilidade. Não há, contudo, informação sobre o efeito da in- teração na seleção precoce, na cultura do feijoeiro. Entretanto, em trigo ela foi relatada por O'BRIEN et al. (1978) e WHAN et al. (1982).

As populações segregantes utilizadas podem apresentar diferentes freqüências alélicas, advindas de cruzamentos envolvendo mais de dois genitores ou de cruzamentos em que participam em diferentes proporções. Não há informações de que a eficiência da seleção precoce varia com a freqüência alélica da população segregante.

Os objetivos deste trabalho foram avaliar a eficiência da seleção precoce para a produtividade de grãos de feijão, utilizando o método de bulk dentro de famílias $F_{2}$, e fornecer informações a respeito das possíveis implicações da interação na seleção precoce.

\section{MATERIAL E MÉTODOS}

Este trabalho foi conduzido no Campus da Universidade Federal de Lavras (UFLA), em Lavras (MG), onde foram avaliadas famílias segregantes (feijoeiros), oriundas do cruzamento entre os cultivares Carioca $\left(\mathrm{P}_{1}\right)$ e FT-Tarumã $\left(\mathrm{P}_{2}\right)$. O genitor Carioca possui hábito de crescimento tipo III, apresenta grãos pequenos de tegumento bege com estrias marrons e é proveniente do Instituto Agronômico de Campinas (SP). O genitor FT-Tarumã é oriundo da FT-Pesquisa e Sementes, possui hábito de crescimento tipo II, grãos pequenos de cor preta. Os cruzamentos foram realizados em casa de vegetação, em 1995. Posteriormente foram obtidas as gerações $F_{2}, F_{2} R_{1}$ e $F_{2} R C_{2}$, sob condições de campo, quando então colheram-se, aleatoriamente, plantas individuais que deram origem às famílias, as quais foram avançadas no campo por meio do método bulk dentro de famílias $\mathrm{F}_{2}$.

Foram obtidas 64 famílias derivadas de plantas da geração $F_{2}$, 64 de $F_{2} R C_{1}$ e 64 do $F_{2} R_{2}$, totalizando 192 famílias, avaliadas juntamente com os dois genitores e duas testemunhas (Carioca MG e Pérola), na geração $\mathrm{F}_{2: 3}$, utilizando-se o delineamento látice simples $14 \mathrm{x}$ 14. As parcelas foram constituídas de uma linha de um metro, semeada na safra da seca (fevereiro de 1997). A densidade de semeadura foi de 15 sementes. $\mathrm{m}^{-1}$.

Foi realizada a adubação no momento da semeadura com o equivalente a $400 \mathrm{~kg}$.ha ${ }^{-1}$ da fórmula 4-14-8 de N, $\mathrm{P}_{2} \mathrm{O}_{5}$ e $\mathrm{K}_{2} \mathrm{O}$ e aplicado em cobertura o equivalente a $150 \mathrm{~kg}$.ha ${ }^{-1}$ de sulfato de amônio, 25 dias após a semeadura. A única aplicação de defensivo 
agrícola foi feita utilizando o inseticida Phorate no momento da semeadura. Quando houve deficiência hídrica a cultura recebeu irrigação suplementar por aspersão. A característica avaliada foi a produção de grãos, em g.parcela ${ }^{-1}$.

As famílias foram novamente avaliadas durante mais três gerações: $F_{2: 4}$, semeadura no inverno (julho de 1997); $F_{2: 5}$, semeadura nas águas (novembro de 1997); e $F_{2: 6}$, semeadura na seca (fevereiro de 1998). Para as avaliações adotou-se o mesmo delineamento e os mesmos tratos culturais, com exceção da parcela, que foi de duas linhas de dois metros.

Os dados relativos à produção de grãos em kg.ha ${ }^{-1}$, foram submetidos à análise de variância individual; procedeu-se, em seguida, à análise conjunta envolvendo todas as gerações, utilizando-se as médias ajustadas. Antes da análise conjunta, porém, foi efetuado o teste de homogeneidade de variância. Em razão da não-ocorrência de homocedasticidade foi utilizado o método de Cochran, citado por PIMENTEL-Gomes (1985).

A partir das análises de variância foram estimados os componentes de variância genética, variância fenotípica, herdabilidade no sentido amplo, e variância da interação famílias $x$ gerações, sendo, a última, decomposta em parte simples e complexa, utilizando- se procedimento de VeNCOvSKY (1987). Também foi estimada a correlação genética entre o desempenho médio das famílias nas gerações sucessivas. A estimativa da herdabilidade realizada foi obtida utilizandose a expressão semelhante à apresentada por FEHR (1987), ou seja: $\mathrm{h}_{\mathrm{ij}}^{2}=\frac{G S_{\mathrm{j}} / \mathrm{m}_{\mathrm{j}}}{\mathrm{ds} \mathrm{s}_{\mathrm{i}} / \mathrm{m}_{\mathrm{i}}}$, em que: $G S_{\mathrm{j}}$ é o desempenho na geração $\mathrm{j}$ das famílias selecionadas na geração $i$, menos a média geral dos indivíduos da geraçãoj; $d s_{i}$ é o diferencial de seleção, ou seja, a média dos indivíduos selecionados na geração i menos a média geral dos indivíduos dessa geração; e $m_{i}$ e $m_{j}$ são as médias das famílias selecionadas nas gerações i e j respectivamente. Para fins de cálculo considerouse a seleção das 20 famílias, isto é, uma intensidade de seleção simulada de $10,4 \%$.

Foi também estimado o ganho com a seleção divergente utilizando-se a seguinte expressão:

$G S(\%)=\left[\left(\frac{A-B}{B}\right) \times 100\right]$, em que: A é a média das 20 famílias mais ou menos produtivas, selecionadas na geração i e avaliadas na geração j; e B é a média geral das famílias avaliadas na geração $j$.

Além disso, estimou-se a correlação entre o desempenho fenotípico das famílias na geração i e o desempenho genotípico na geração $\mathrm{j}\left(\mathrm{r}_{\mathrm{FiGj}}\right)$, pela ex-

Quadro 1. Resumo da análise de variância conjunta para o caráter produtividade de grãos (kg.ha $\left.{ }^{-1}\right)$, obtida na avaliação das famílias das gerações $F_{2: 3}, F_{2: 4,} F_{2: 5}$ e $F_{2: 6}$ do cruzamento Carioca x FT-Tarumã, em Lavras (MG), 1997/1998

\begin{tabular}{lcc}
\hline FV & GL & QM \\
Gerações (E) & 3 & $331582464,20^{* *}$ \\
Tratamentos $(G)$ & 195 & $843533,00^{* *}$ \\
$\quad$ Entre famílias F2 & 63 & $701614,07^{*}$ \\
Entre famílias RC 1 & 63 & $737309,92^{*}$ \\
Entre famílias RC2 & 63 & $1017048,64^{* *}$ \\
Entre tipos de famílias & 2 & $3604324,50^{* *}$ \\
Entre testemunhas & 3 & 640335,15 \\
Testemunhas vs. Famílias & 1 & 633052,00 \\
Interação (GE) & $420^{(1)}(585)$ & $4932004,06^{* *}$ \\
Entre famílias F2 ${ }_{2}$ Gerações & $135(189)$ & $504957,52^{*}$ \\
Entre famílias RC ${ }_{x}$ Gerações & $135(189)$ & $561274,09^{* *}$ \\
Entre famílias RC ${ }_{x}$ Gerações & $135(189)$ & 410775,08 \\
Entre tipos de famílias ${ }_{x}$ Gerações & $4(6)$ & $1047964,66^{*}$ \\
Entre testemunhas ${ }_{x}$ Gerações & $6(9)$ & 106977,93 \\
Testemunhas vs. famílias ${ }_{x}$ Gerações & $2(3)$ & 708276,11 \\
Erro médio & $425(676)$ & 392395,19 \\
Média & & 2719,82 \\
CV\% & & 23,03 \\
\hline
\end{tabular}

${ }^{(1)}$ Graus de liberdade estimados pelo método de Cochran (PIMENTEEGOMES, 1985).

, ${ }^{* *}$ Significativo a $5 \%$ e $1 \%$ de probabilidade respectivamente. 
Quadro 2. Produtividade média de grãos de feijão, kg.ha ${ }^{-1}$, de famílias do cruzamento Carioca x FT-Tarumã avaliadas nas diferentes gerações, em Lavras (MG), 1997/1998

\begin{tabular}{|c|c|c|c|}
\hline Gerações & Tipo & $\begin{array}{c}\text { Produtividade } \\
\text { Média }\end{array}$ & Variação \\
\hline \multirow{7}{*}{$\mathrm{F}_{2: 3}$} & F2 & 3609 & $2127-5011$ \\
\hline & $\mathrm{F}_{2} \mathrm{RC}_{1}$ & 3709 & $2638-5032$ \\
\hline & $\mathrm{F}_{2} \mathrm{RC}_{2}$ & 2928 & $2092-5851$ \\
\hline & Média & 3415 & \\
\hline & Pais: Carioca & 2929 & \\
\hline & FT-Tarumã & 3350 & \\
\hline & Testemunhas & 4158 & \\
\hline \multirow{7}{*}{$\mathrm{F}_{2: 4}$} & F2 & 2772 & $1176-4352$ \\
\hline & $\mathrm{F}_{2} \mathrm{RC}_{1}$ & 2779 & $1512-3913$ \\
\hline & $\mathrm{F}_{2} \mathrm{RC}_{2}$ & 2931 & $2112-4331$ \\
\hline & Média & 2827 & \\
\hline & Pais: Carioca & 2930 & \\
\hline & FT-Tarumã & 3351 & \\
\hline & Testemunhas & 3248 & \\
\hline \multirow{7}{*}{$F_{2: 5}$} & F2 & 1498 & $873-2301$ \\
\hline & $\mathrm{F}_{2} \mathrm{RC}_{1}$ & 1558 & $830-2153$ \\
\hline & $\mathrm{F}_{2} \mathrm{RC}_{2}$ & 1445 & $838-2014$ \\
\hline & Média & 1500 & \\
\hline & Pais: Carioca & 1953 & \\
\hline & FT-Tarumã & 1872 & \\
\hline & Testemunhas & 1968 & \\
\hline \multirow{7}{*}{$F_{2: 6}$} & $\mathrm{~F} 2$ & 2684 & $1926-3648$ \\
\hline & $\mathrm{F}_{2} \mathrm{RC}_{1}$ & 2765 & $1913-3873$ \\
\hline & $\mathrm{F}_{2} \mathrm{RC}_{2}$ & 2924 & $2041-3953$ \\
\hline & Média & 2791 & \\
\hline & Pais: Carioca & 2396 & \\
\hline & FT-Tarumã & 2604 & \\
\hline & Testemunhas & 2839 & \\
\hline \multirow{7}{*}{$\begin{array}{l}\text { M é d i a } \\
\text { Geral }\end{array}$} & $\mathrm{F}_{2}$ & 2641 & $2066-3249$ \\
\hline & $\mathrm{F}_{2} \mathrm{RC}_{1}$ & 2703 & $2240-3689$ \\
\hline & $\mathrm{F}_{2} \mathrm{RC}_{2}$ & 2807 & $2110-3741$ \\
\hline & Média & 2633 & \\
\hline & Pais: Carioca & 2552 & \\
\hline & FT-Tarumã & 2794 & \\
\hline & Testemunhas & 3053 & \\
\hline
\end{tabular}

pressão: $r_{\mathrm{FiGj}}=\mathrm{r}_{\mathrm{GiGj}} \sqrt{\mathrm{h}_{\mathrm{i}}^{2}}$, em que: $\mathrm{r}_{\mathrm{GiGj}}$ é a correlação genética e que é função apenas do coeficiente de endogamia (I) nas gerações consideradas, isto é, $\mathrm{r}_{\mathrm{GiGj}}=\sqrt{\left(1+\mathrm{I}_{\mathrm{i}}\right) /\left(1+\mathrm{I}_{\mathrm{j}}\right)} ; \mathrm{h}_{\mathrm{i}}^{2}$ é a herdabilidade do caráter na geração i, quando é efetuada a seleção (BERNARDO, 1991).

\section{RESULTADOS E DISCUSSÃO}

Ocorreu diferença significativa entre as fontes de variação:gerações, tratamentos e interação tratamentos $x$ gerações e entre as famílias derivadas de plantas da geração $\mathrm{F}_{2}$ e de ambos os retrocruzamentos (Quadro 1). Analogamente, detectou-se diferença significativa também no desempenho médio dos três tipos de famílias. Contudo, não se constatou significância para o teste $\mathrm{F}$ entre as testemunhas e entre $\mathrm{o}$ desempenho médio das testemunhas versus as 192 famílias avaliadas.

$\mathrm{Na}$ decomposição da variação tratamentos $x$ gerações, observou-se que ela só foi significativa quando envolveu as famílias derivadas das plantas da geração $\mathrm{F}_{2}$ e $\mathrm{RC}_{1}$ e entre os tipos de progênies. Destaca-se o fato de que entre as famílias derivadas do $\mathrm{RC}_{2}$, ainda que sempre fosse detectada diferença significativa, o comportamento foi coincidente nas sucessivas gerações, evidenciando que esse tipo de família não interagiu com o ambiente.

A existência de variabilidade entre as famílias pode ser constatada pela grande variação observada na produtividade de grãos. Embora a produtividade média das famílias não tenha diferido das testemunhas, varificou-se grande variação entre as famílias, tendo algumas apresentado desempenho superior à média das testemunhas (Quadro 2).

No presente trabalho foram avaliadas famílias com diferentes contribuições dos alelos dos dois genitores envolvidos. No caso das famílias derivadas de $\mathrm{F}_{2}$, a contribuição dos pais é idêntica, ou seja, $50 \%$ de cada um. Nas famílias de $\mathrm{RC}_{1}, 75 \%$ dos alelos são provenientes do cultivar Carioca $\left(\mathrm{P}_{1}\right)$ e $25 \%$ do FTTarumã $\left(\mathrm{P}_{2}\right)$. Nas derivadas de $\mathrm{RC}_{2}$ ocorreu o inverso. Em termos da produtividade média, o melhor desempenho ocorreu entre as famílias derivadas de $\mathrm{RC}_{2} \mathrm{e}, \mathrm{o}$ pior, na $\mathrm{F}_{2}$. Entretanto, a diferença não se acentuou em demasia, visto que a superioridade da média de $\mathrm{RC}_{2}$ em relação a $F_{2}$ foi de $6,8 \%$ (Quadro 2). Isso pode ser explicado se considerarmos que, exceto nas avaliações envolvendo as famílias $\mathrm{F}_{2: 5}$, $\mathrm{o}$ desempenho do cultivar FT-Tarumã utilizado como testemunha foi superior ao Carioca. Assim, a maior proporção dos alelos daquele cultivar pode ter contribuído para o melhor desempenho das famílias de $\mathrm{RC}_{2}$. É importante enfatizar que, entre as 20 melhores famílias na geração $F_{2: 6}, 11$ são provenientes de $R_{2}, 5$ de $R_{1}$ e 4 de $\mathrm{F}_{2}$.

O relato de ocorrência de interação famílias $\mathrm{x}$ ambientes é freqüente, na literatura, com a cultura do 
Quadro 3. Estimativas dos componentes da variância genética $\left(\hat{\sigma}_{\mathrm{G}}^{2}\right)$, da variância da interação famílias $\mathrm{x}$ gerações $\left(\hat{\sigma}_{\mathrm{GE}}^{2}\right)$ e decomposição em parte simples e complexa e estimativas das correlações genéticas ( $\mathrm{r}_{\mathrm{G}}$ ), para a produtividade de grãos (kg.ha-1), obtidas na avaliação das famílias do cruzamento Carioca x FT-Tarumã, considerando as diferentes gerações, duas a duas, em Lavras (MG), 1997/1998

\begin{tabular}{lrrrrrrr} 
Gerações & & & & \multicolumn{2}{c}{ Decomposição de $\hat{\sigma}_{\mathrm{GE}}^{2}$} \\
\cline { 6 - 7 } & $\hat{\sigma}_{\mathrm{G}}^{2}$ & $\hat{\sigma}_{\mathrm{GE}}^{2}$ & $\hat{\sigma}_{\mathrm{GE}}^{2} / \hat{\sigma}_{\mathrm{G}}^{2}$ & Parte Simples & Parte Complexa & rG \\
$\mathrm{F}_{2: 3} \mathrm{~F}_{2: 4}$ & 104683,03 & 28945,52 & 0,28 & 9014,54 & 19930,98 & 0,84 \\
$\mathrm{~F}_{2: 3} \mathrm{~F}_{2: 5}$ & 8541,17 & 102353,82 & 11,98 & 25759,58 & 76594,24 & 0,10 \\
$\mathrm{~F}_{2: 3} \mathrm{~F}_{2: 6}$ & 66371,21 & 64281,17 & 0,97 & 10457,28 & 53823,89 & 0,55 \\
$\mathrm{~F}_{2: 4} \mathrm{~F}_{2: 5}$ & 22939,93 & 39591,11 & 1,72 & 4232,17 & 35358,94 & 0,39 \\
$\mathrm{~F}_{2: 4} \mathrm{~F}_{2: 6}$ & 34977,65 & 47310,94 & 1,35 & 26,11 & 47284,83 & 0,42 \\
$\mathrm{~F}_{2: 5} \mathrm{~F}_{2: 6}$ & 24734,73 & 34820,35 & 1,41 & 3358,31 & 31462,04 & 0,44 \\
\hline
\end{tabular}

feijoeiro (TAKEDA et al., 1991; ABREU et al., 1990; GONÇALVES, 1995). Quando se estuda a interação, o importante não é apenas estimar sua magnitude mas, sobretudo, verificar sua causa (VENCOVSKY, 1987), ou seja, verificar se ela é de natureza simples, devido à diferença na manifestação genotípica nos diferentes ambientes, ou se é complexa, advinda de um comportamento não coincidente nos ambientes avaliados. Visando elucidar melhor a contribuição da interação, procedeu-se à análise de variância das gerações, aos pares. Verificou-se que a contribuição da variância genética da interação oscilou acentuadamente, a saber: 0,28 vezes o valor da $\hat{\sigma}_{\mathrm{G}}^{2}$ no par $\mathrm{F}_{2: 3} / \mathrm{F}_{2: 4} \mathrm{e}, 11,98$ vezes, na análise envolvendo as gerações $F_{2: 3} / F_{2: 5}$ (Quadro 3). A decomposição do componente da interação $\left(\hat{\sigma}_{\mathrm{GE}_{\mathrm{H}}}^{2}\right)$ em parte simples e complexa revela que houve predominância da parte complexa em todos os casos (Quadro 3). Em tal situação, é esperado que esta afete a eficiência da seleção precoce, visto indicar que o desempenho relativo das famílias é modificado com as alterações de ambientes.

Na maioria dos trabalhos apresentados na literatura, visando avaliar a eficiência da seleção precoce, o parâmetro utilizado é a estimativa da correlação genética $\left(\mathrm{r}_{\mathrm{G}}\right)$ entre o desempenho das famílias, na geração i e $\mathrm{i}+\mathrm{x}$, em que $\mathrm{x}$ pode assumir o valor 1,2 ou 3 , ou, às vezes, até um número maior de gerações. Pelos resultados apresentados no quadro 3, verificase que a $r_{G}$ foi maior quando envolveu as gerações $\mathrm{F}_{2: 3} / \mathrm{F}_{2: 4}\left(\mathrm{r}_{\mathrm{G}}=0,84\right)$ e de menor magnitude no par $\mathrm{F}_{2: 3} / \mathrm{F}_{2: 5}\left(\mathrm{r}_{\mathrm{G}}=0,10\right)$. Contudo, em relação à última geração avaliada, $\mathrm{F}_{2: 6}$, a correlação equivaleu a 0,55 $\left(\mathrm{F}_{2: 3} / \mathrm{F}_{2: 6}\right)$.

Há, na literatura, estimativas da correlação do desempenho entre famílias em gerações sucessivas, considerando a produtividade de grãos que são inferiores às relatadas neste trabalho. Com a cultura da soja, KNotT e KumaR (1975) estimaram a correlação entre famílias $\mathrm{F}_{2: 3}$ e $\mathrm{F}_{2: 5}$ encontrando valores que oscilaram entre 0,14 e 0,29. Em relação ao trigo, WHAN et al. (1981) estimaram a correlação entre famílias $\mathrm{F}_{2: 3}$ e $\mathrm{F}_{2: 5}$, tendo obtido valores entre 0,10 e 0,49 . No caso específico do feijoeiro, RANALLI et al. (1996) estimaram a correlação entre famílias $F_{2: 3}$ e $F_{2: 5}$, tendo obtido $r$ de 0,20 a 0,25 .

Outra maneira de se avaliar a eficiência da seleção precoce é através da estimativa da herdabilidade. Observa-se que, de modo geral, as estimativas foram novamente altas, exceto quando se considerou a geração $F_{2: 3}$ e o ganho em $F_{2: 5}$ ou $F_{2: 6}$ (Quadro 4).

Dado que as famílias foram avaliadas simultaneamente, em todas as gerações, foi possível avaliar a seleção precoce por meio da seleção divergente, isto é, foram identificadas as 20 famílias mais produtivas e as 20 menos produtivas, nas gerações mais precoces (i), e acompanhar o desempenho das mesmas nas gerações sucessivas (j). A seleção efetuada em $F_{2: 3}$ contribuiu para um ganho de $3,5 \%$ na geração $F_{2: 6}$, no sentido de aumentar a produtividade, e de $-5,3 \%$ para a redução na expressão do caráter. Quando a seleção foi efetuada em $\mathrm{F}_{2: 4}$ o ganho, especialmente no sen-

Quadro 4. Estimativas da herdabilidade realizada (\%) considerando a seleção na geração i e a resposta na geração j, do cruzamento Carioca x FT-Tarumã em Lavras (MG), $1997 / 1998$

\begin{tabular}{lccc} 
& \multicolumn{3}{c}{ Geraçãoj } \\
\cline { 2 - 4 } Geração i & $\mathrm{F}_{2: 4}$ & $\mathrm{~F}_{2: 5}$ & \multicolumn{1}{c}{$\mathrm{F}_{2: 6}$} \\
\hline $\mathrm{F}_{2: 3}$ & 31,00 & 0,00 & 9,55 \\
$\mathrm{~F}_{2: 4}$ & & 26,00 & 31,00 \\
$\mathrm{~F}_{2: 5}$ & & & 16,00 \\
\hline
\end{tabular}


Quadro 5. Médias em kg.ha ${ }^{-1}$ das 20 famílias mais produtivas (Prod +) e das 20 menos produtivas (Prod-) nas gerações de seleção i, e desempenho das mesmas famílias nas gerações sucessivas j, do cruzamento Carioca x FT-Tarumã, em Lavras (MG), 1997/1998

\begin{tabular}{|c|c|c|c|c|c|c|}
\hline \multirow{3}{*}{$\begin{array}{l}\text { Geração de } \\
\text { Seleção (i) }\end{array}$} & \multicolumn{6}{|c|}{ Desempenho das famílias na Geração j } \\
\hline & \multicolumn{2}{|c|}{$\mathrm{F}_{2: 4}$} & \multicolumn{2}{|c|}{$\mathrm{F}_{2: 5}$} & \multicolumn{2}{|c|}{$\mathrm{F}_{2: 6}$} \\
\hline & Prod+ & Prod- & Prod + & Prod - & Prod + & Prod - \\
\hline $\mathrm{F}_{2: 3}$ & 3146 & 2654 & 1496 & 1460 & 2888 & 2641 \\
\hline GS $(\%)$ & $(11,28)^{(1)}$ & $(-6,12)$ & $(-0,27)$ & $(-2,67)$ & $(3,47)$ & $(-5,37)$ \\
\hline $\mathrm{F}_{2: 4}$ & & & 1636 & 1448 & 3087 & 2642 \\
\hline GS $(\%)$ & & & $(9,06)$ & $(-3,47)$ & $(10,60)$ & $(-5,34)$ \\
\hline $\mathrm{F}_{2: 3}+\mathrm{F}_{2: 4}$ & & & 1566 & 1385 & 2995 & 2454 \\
\hline GS $(\%)$ & & & $(4,4)$ & $(-7,66)$ & $(7,35)$ & $(-12,07)$ \\
\hline $\mathrm{F}_{2: 5}$ & & & & & 2954 & 2762 \\
\hline GS (\%) & & & & & $(5,84)$ & $(-1,04)$ \\
\hline $\mathrm{F}_{2: 6}$ & & & & & 3587 & 2042 \\
\hline GS (\%) & & & & & $(28,52)$ & $(-26,84)$ \\
\hline Média Geral & \multicolumn{2}{|c|}{2827} & \multicolumn{2}{|c|}{1500} & \multicolumn{2}{|c|}{2791} \\
\hline
\end{tabular}

${ }^{(1)} \mathrm{GS}(\%)=$ [(média das 20 famílias mais produtivas ou menos produtivas, selecionadas na geração i, avaliadas na geração j-média geral das famílias avaliadas na geração j) (média geral das famílias da geração j) x 100].

tido de aumento de produtividade, foi mais expressivo, ou seja, superior a $9 \%$. Verificou-se também que os ganhos mostraram-se inferiores sempre que o processo envolveu a geração $\mathrm{F}_{2: 5}$, a qual, provavelmente, mais contribuiu para a interação (Quadro 5). Em face da ocorrência de interação famílias $x$ gerações, uma das formas de se aumentar a eficiência da seleção precoce seria através da seleção efetuada em duas ou mais gerações simultâneas. Esse fato é comprovado pelo ganho observado em $\mathrm{F}_{2: 6}$, quando a seleção foi efetuada na média das gerações $\mathrm{F}_{2: 3}+\mathrm{F}_{2: 4}$.

A eficiência da seleção precoce também pode ser avaliada pela estimativa da correlação entre $o$ fenótipo do indivíduo ou da família, na geração i, e o genótipo, na geração j (BERNARDO, 1991). No quadro 6 observa-se que foi superior a $50 \%$, em todos os casos,

Quadro 6. Estimativas das correlações entre os valores fenotípicos das famílias na geração precoce i, e o valor genético na geração avançada $\mathrm{j}$, segundo o método de Bernardo (1991).UFLA-Lavras (MG), 1997/1998

\begin{tabular}{llcccc} 
& & \multicolumn{4}{c}{ Geração j } \\
\cline { 3 - 6 } Geração i & $\mathrm{I}^{(1)}$ & $\mathrm{F}_{2: 4}$ & $\mathrm{~F}_{2: 5}$ & $\mathrm{~F}_{2: 6}$ & $\mathrm{~F} 2: \infty_{\infty}$ \\
\hline $\mathrm{F}_{2: 3}$ & 0,5 & 50,03 & 47,87 & 47,34 & 47,00 \\
$\mathrm{~F}_{2: 4}$ & 0,75 & & 56,26 & 55,11 & 53,95 \\
$\mathrm{~F}_{2: 5}$ & 0,875 & & & 65,58 & 64,91 \\
$\mathrm{~F}_{2: 6}$ & 0,9375 & & & & 61,66 \\
\hline
\end{tabular}

(1)I: Coeficiente de endogamia. exceto quando envolveu a geração $\mathrm{F}_{2: 3}$ e considerouse a resposta a partir de $F_{2: 5}$. Esse resultado evidencia que a seleção precoce avaliando-se famílias derivadas do método bulk dentro de famílias F2, foi eficiente. No entanto é válido mencionar que a herdabilidade, quando a seleção é efetuada em apenas um ambiente, é superestimada, pois, como mencionado anteriormente, as estimativas dependem principalmente da herdabilidade do caráter, no momento da seleção.

\section{CONCLUSÕES}

1. A maior freqüência de famílias com desempenho superior foi observada na população com $75 \%$ dos alelos do cultivar FT- Tarumã, provavelmente devido à superioridade desse cultivar.

2. A seleção precoce foi eficiente, sobretudo na eliminação das famílias com pior desempenho, reduzindo, assim, o trabalho dos melhoristas em gerações avançadas.

3. A interação genótipos $x$ ambientes afeta a seleção precoce; visando melhorar sua eficiência, as famílias devem ser avaliadas durante pelo menos duas gerações.

\section{REFERÊNCIAS BIBLIOGRÁFICAS}

ABREU, A.F.B.; RAMALHO, M.A.P.; SANTOS, J.B. dos; PEREIRA FILHO, I.A. Effects genotype x environment interaction on estimations of genetic and phenotypic parameters of commom beans. Revista Brasileira de Genética, Ribeirão Preto, v.13, n1, p.75-82, 1990. 
BERNARDO, R. Correlation between tests cross performance of lines at early and last selfing generation. Theoretical Applied Genetics, Berlin, v. 82, p.17-21, 1991.

CUTRIM, V. dos A.; RAMALHO, M.A.P.; CARVALHO, A.M. Eficiência da seleção visual na produtividade de grãos de arroz (Oryza sativa L.) irrigado. Pesquisa Agropecuária Brasileira, Brasília, v.32, n.6, p 601-606, 1997.

FEHR, W. R. Principles of cultivar development: theory and technique. New York: Macmillan Publishing, 1987, v.1.

GONÇALVES, P.R. Obtenção de linhagens de feijoeiro (Phaseolus vulgaris L.) com grãos tipo carioca e resistente à antracnose e mancha angular. Lavras, 1995. 65p. Dissertação (Mestrado em Genética e Melhoramento de Plantas) - UFLA.

HANSON, W.D.; LEFFIL, R.C.; JOHNSON, H.W. Visual discrimination for yield among soybean phenotypes. Crops Science, Madison, v.2, n.2, p.93-96, 1962.

KNOTT, D. R.; KUMAR, J. Comparison of early generation yield testing and a single seed descent procedure in wheat breeding. Crop Science, Madison, v.15, n.3, p.295-299, 1975.

O'BRIEN, L.; BAKER, R.J.; EVANS, L.E. Response to selection for yield in $\mathrm{F}_{3}$ of four wheat cross. Crop Science, Madison, v.18, n.6, p.1029-1033, 1978.

PATIÑO, H.; SINGH, S. P. Respuesta a la selección visual para rendimiento en generaciones $F_{2}$ y $F_{3}$ en frijol, Phaseolus vulgaris L. Cali: CIAT, 1989. p.417. (CIAT Documento, 47).
PIMENTEL-GOMES, F. Curso de estatística experimental. 5.ed. Piracicaba: Nobel, 1985. 466p.

RAMALHO, M.A.P.; SANTOS, J.B. dos; ZIMMERMANN, M.J.O. Genética quantitativa em plantas autógamas: aplicações no melhoramento do feijoeiro. Goiânia:UFG, 1993. 271p.

RANALLI, P.; RUARO, G.; DEL RE, P.; FAETI, V. Comparason of early generation yield testing and a single seed descent procedure in two bean (Phaseolus vulgaris L.) crosses. Journal Genetics E Breeding, Italy, v.50, n.2, p.103-108, 1996.

TAKEDA, C.; SANTOS, J.B. dos; RAMALHO, M.A.P. Choice of parental lines for common bean (Phaseolus vulgaris L.) breeding. II. Reaction of cultivars and of their segregant populations to variations in different environments. Revista Brasileira de Genética, Ribeirão Preto, v.14, n.2, p.455-465, 1991.

VENCOVSKY, R. Herança quantitativa. In: PATTERNIANI, E.; VIÉGAS, G. P. (Eds). Melhoramento e produção de milho. 2 ed., Campinas: Fundação Cargill, 1987. v.1, cap.5, p. 137-214.

WHAN, B.R.; RATHJEN, A.J.; KNIGHT, R.R. The relation between wheat lines derived from then $\mathrm{F}_{2}, \mathrm{~F}_{3}, \mathrm{~F}_{4}$, and $\mathrm{F}_{5}$ generations for grain yield and harvest index. Euphytica, Wageningen, v.30, n.2, p.419-430, 1981.

WHAN, B.R.; RATHJEN, A.J.; KNIGHT, R.R. Response to selection for grain yield and harvest index in $\mathrm{F}_{2}, \mathrm{~F}_{3}$ and $\mathrm{F}_{4}$ derived lines of two wheat crosses. Euphytica, Wageningen, v.31, p 139-150, 1982. 